\title{
Association of Migraine and Ischemic Heart Disease: A Review
}

\author{
Aisha Saeed $^{1}$, Kiran F. Rana ${ }^{1}$, Zain I. Warriach ${ }^{2}$, Muhammad Ali Tariq ${ }^{3}$, Bilal Haider Malik ${ }^{3}$ \\ 1. Family Medicine, California Institute of Behavioral Neurosciences and Psychology, Fairfield, USA 2. Psychiatry, \\ California Institute of Behavioral Neurosciences and Psychology, Fairfield, USA 3. Internal Medicine, California \\ Institute of Behavioral Neurosciences and Psychology, Fairfield, USA
}

Corresponding author: Aisha Saeed, aisha.saeed1310@gmail.com

\begin{abstract}
Many new studies have shown an association between migraine and ischemic heart disease, and the association seems to be multi-factorial. This article reviews what is already known about this linkage and further investigates if migraine is a risk factor for cardiovascular disease.
\end{abstract}

The literature search for this article was performed primarily using PubMed as the search engine. Only those articles that assessed migraine as exposure and cardiovascular events as outcomes were included. Also, articles only from the last five years with full-text and human studies were reviewed.

Based on our investigation and as indicated by previous studies, migraine headache is associated with different kinds of cardiovascular events. Healthcare providers need to be aware of this association so that they can assess and manage their migraine patients better.

Categories: Family/General Practice, Internal Medicine, Neurology

Keywords: migraine, headache, cardiovascular diseases, ischemic heart disease, myocardial ischemia, migraine disorders, coronary artery disease, migraine and ischemic heart disease

\section{Introduction And Background}

The healthcare cost associated with migraine is about $\$ 36$ billion annually in the United States, which is expected to rise in the future. While the total expenditure on cardiovascular disease was $\$ 555$ billion in the US in the year 2016, and this figure is projected to go up to $\$ 1.1$ trillion by 2035 .

Migraine is a neurological disease affecting approximately one billion people worldwide and is considered as the 6th most disabling disease in the world [1-3]. It is more common in women than men and affects over $30 \%$ of women between the ages of 25 and 55 [4,5]. Due to the throbbing nature of the disease, it is common to think of migraines to have a vascular etiology, when in fact there are three different theories including vascular theory, neurovascular theory and cortical spreading depolarization (CSD) [6,7]. Migraine is an

Received 08/28/2019

Review began 09/09/2019 Review ended 09/15/2019 Published 09/21/2019

\section{() Copyright 2019}

Saeed et al. This is an open access article distributed under the terms of the Creative Commons Attribution License CC-BY 3.0., which permits unrestricted use, distribution, and reproduction in any medium, provided the original author and source are credited. inherent disease having genetic association of about $42 \%$ [8]. Certain environmental factors can bring about or worsen a migraine attack including diet, stress, alcohol, smoking, sleep disturbance, hormonal changes, sensory stimuli (light smells or noises), weather change and certain foods $[9,10]$.

Numerous studies have shown that migraineurs have a higher chance of experiencing an ischemic stroke, a risk two times more common in patients experiencing migraine with aura. Aura is a reversible set of neurologic symptoms that usually comes before a migraine attack, is mostly visual and lasts about 5-60 minutes. Aura is only seen in $1 / 4$ th of the population suffering from migraine [11,12].

Migraine (aura) can be considered one of the risk factors for ischemic heart disease (IHD) according to new emerging evidence $[6,13]$. Although the exact relationship between migraine and ischemic heart disease is still not completely known, some studies suggest the association to be multi-factorial, suggesting different mechanisms for different cardiovascular events [14]. One of the proposed mechanisms seems to be endothelial dysfunction [15-17].

A higher prevalence of patent foramen ovale in patients suffering from migraine (aura), increased release of inflammatory markers, and the use of non-steroid anti-inflammatory drugs (NSAID) in these patients can further increase the likelihood of myocardial infarction, venous thromboembolism and atrial fibrillation [1822]. Some studies also suggest the association between migraine and cardiovascular events to be genetic in origin, as shown by a genome-wide analysis study [23-25].

There is an urgent need to understand the association between migraine and ischemic heart disease (IHD) so that improved adjustments can be made in the treatment of patients suffering from migraine. In this review, we are going to investigate further the population most at risk of cardiovascular events with migraine headache. This article will help guide physicians to manage patients better and decide what preventive 


\section{Cureus}

strategies need to be used to minimize the risk of future cardiovascular events in migraineurs.

\section{Review}

\section{Methods}

The electronic search was performed mostly using PubMed as the search engine. Some articles were also taken from Google Scholar, Embase, MEDLINE, and grey literature. The search was performed using the Mesh terms and keywords "Migraine”, "Ischemic Heart Disease (IHD)", "Cardiovascular disease (CVD)", "Migraine and IHD”, "Headache and Cardiovascular disease”, "Myocardial ischemia”, "Migraine disorder” and "Coronary artery disease". The number of articles yielded with each keyword and mesh term is shown in Tables 1,2. No guidelines or statements were taken from preferred reporting items for systematic reviews and meta-analyses (PRISMA) or meta-analyses of observational studies in epidemiology (MOOSE).

\begin{tabular}{|c|c|c|}
\hline KEYWORDS & DATABASE & NUMBER OF RESULTS \\
\hline Cardiovascular Disease & PubMed & 2417123 \\
\hline Ischemic Heart Disease & PubMed & 486120 \\
\hline Headache & PubMed & 91338 \\
\hline Migraine & PubMed & 37409 \\
\hline Headache and cardiovascular disease & PubMed & 16616 \\
\hline Migraine and Ischemic Heart Disease & PubMed & 592 \\
\hline
\end{tabular}

TABLE 1: Search results with simple keywords

\begin{tabular}{|c|c|c|}
\hline MeSH KEYWORDS & DATABASE & NUMBER OF RESULTS \\
\hline Myocardial Ischemia & PubMed & 470064 \\
\hline Migraine Disorder & Google Scholar & 291000 \\
\hline Coronary Artery Disease & PubMed & 160695 \\
\hline
\end{tabular}

TABLE 2: Search results with mesh keywords

All types of studies and review articles evaluating cardiovascular outcomes in migraine patients were included. Other selection criteria used in this review were: full-text articles, articles published in the last five years, and articles with humans as subjects. Some facts were also taken from grey literature.

Everything was done scientifically and ethically. We did not use any quality assessment tool or perform any statistical analyses.

\section{Results}

The initial search was performed with PubMed using the following keywords: 'Cardiovascular Disease (CVD)' which generated 2417123 articles, 'Ischemic Heart Disease' brought up 486120 studies, 'Headache' resulted in 91338 while search with the word 'Migraine' resulted in 37409 studies. 'Headache and Cardiovascular Disease' generated 16616 articles, and a search with keyword 'Migraine and Ischemic Heart Disease' generated 592 results.

Of these 592 articles, 513 were excluded based on the inclusion/exclusion criteria (full-text, five-years, and human). Among the 79 studies that were reviewed, 40 were excluded from the revision of titles and abstract, four did not evaluate the outcome of interest, and one article was not available. Thirty-four studies were included in the final analysis. There are about eight full articles. The included studies are from five countries. The search was performed without any language restriction.

One of the included articles is a cohort study done in the Danish population, which studied 51032 migraine patients and 510320 people from the general population matched on age, sex, and calendar year. These 


\section{Cureus}

patients were followed up for 19 years, and the study showed that migraine is positively linked with myocardial infarction [14].

A meta-analysis of 16 cohort studies included 1,152,407 subjects with a follow-up duration of up to 26 years [26]. The included studies were adjusted for age and co-morbidities like hypertension, diabetes, and hyperlipidemia. The subjects were assessed either through questionnaires or hospital records, and the final result showed that migraine with aura is a risk factor for major cardiovascular and cerebrovascular events [26].

Two review articles show a strong association between migraine and cardiovascular events [7,27]. Three studies illustrate the association is higher in women suffering from migraine with aura [16,27,28]. A systemic review and meta-analysis of observational studies indicates that migraine is related to myocardial infarction (MI) and angina [15].

Few studies show inconsistent results; one found heterogeneity among reviewed studies and suggested the idea that migraine with aura increases cardiovascular events [29,30].

We agree with the majority of these articles that there is an association between migraine and IHD (Table 3).

\begin{tabular}{|c|c|c|c|}
\hline AUTHOR & YEAR & COUNTRY & OUTCOME \\
\hline $\begin{array}{l}\text { Sacco et al. } \\
{[15]}\end{array}$ & 2015 & Italy & Migraine is associated with Ml and angina and can be considered as a risk factor for CVD. \\
\hline Alqaqa [7] & 2016 & USA & $\begin{array}{l}\text { There is an increased risk of stroke, angina, arrhythmia, PFO, ASD, and MVP with MA. The } \\
\text { physicians should be aware of modifiable cardiovascular risk factors. }\end{array}$ \\
\hline $\begin{array}{l}\text { Linstra et } \\
\text { al. [27] }\end{array}$ & 2017 & $\begin{array}{l}\text { The } \\
\text { Netherlands }\end{array}$ & $\begin{array}{l}\text { Migraine can be appropriately considered as an overall risk factor for CVD, especially in young } \\
\text { women with aura. }\end{array}$ \\
\hline $\begin{array}{l}\text { Rambarat } \\
\text { et al. [16] }\end{array}$ & 2017 & USA & $\begin{array}{l}\text { Those women who have a history of migraine headache and are showing the signs and } \\
\text { symptoms of ischemia are at risk of CVD. }\end{array}$ \\
\hline $\begin{array}{l}\text { Adelborg et } \\
\text { al. [14] }\end{array}$ & 2018 & Denmark & $\begin{array}{l}\text { Migraine ought to be considered a constant risk factor for most cardiovascular diseases in both } \\
\text { men and women. }\end{array}$ \\
\hline $\begin{array}{l}\text { Mahmoud } \\
\text { et al. [26] }\end{array}$ & 2018 & USA & There is an increased risk of cardiovascular and cerebrovascular events with MA. \\
\hline
\end{tabular}

\section{TABLE 3: Researches correlating migraine and CVD}

MI- Myocardial infarction, CVD- Cardiovascular disease, PFO- Patent foramen ovale, ASD- Arterial septal defect, MVP- Mitral valve prolapse, MAMigraine with aura

Some risk factors are commonly shared between migraine and CVD, as indicated in Figure 1 and explained in detail later in our article.

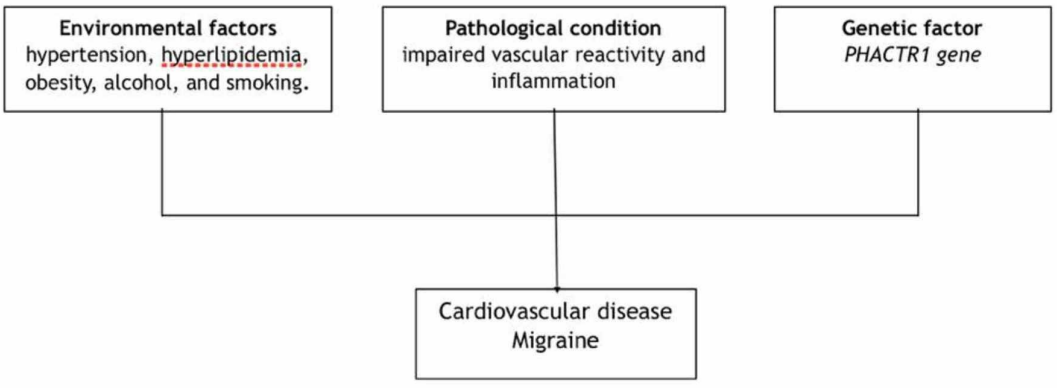

FIGURE 1: Shared risk factors for migraine and CVD

CVD- Cardiovascular disease

PHACTR 1- Phosphatase and actin regulator 1 


\section{Discussion}

The link between migraine and stroke has already been established in literature so we will only highlight the association of migraine and IHD. Our review indicates that migraine is associated with several cardiovascular diseases and this connection is found to be stronger in patients who experience aura than in those without aura. Below are some of the mechanisms involved in the link between migraine and IHD.

Physiological/Pathophysiological Process

To understand the relationship between migraine and cardiovascular events, it is important to understand the mechanisms involved in migraine. Three major theories for the pathophysiology of migraine have been proposed in the literature, including the vascular theory, the neurovascular theory, and the theory of cortical spreading depression (CSD) [7]. The ischemic changes that occur during a migraine attack seem to spread to other organs as well, especially the heart.

The vascular theory suggests that vasoconstriction is responsible for the aura while vasodilatation is responsible for headache during a migraine attack. The neurovascular theory is to be blamed for the neurogenic and vascular alteration in cerebral perfusion that produces migraine episode. Cortical spreading depression can further predispose the brain to cerebral hypoperfusion and arterial ischemia.

Is the proposed pathophysiological mechanism true, or are there other mechanisms causing this association between migraine and IHD?

Biochemical Process

Oxidative stress has also been considered as an important factor in the pathogenesis of migraine headaches and can cause alteration in the cerebral blood flow [31]. A marker for oxidative stress called 4-hydroxy-2nonenal (HNE) has also been found to be elevated in migraineurs and has been suggested to be involved in the pathogenesis of IHD [32]. More importantly, oxidative stress has been associated with endothelial dysfunction, atherosclerosis, and increased vascular risk.

Circulating endothelial progenitor cells (EPC) are responsible for neo-angiogenesis after ischemic insult, and their number and function is found to decline in migraine patients [17,31]. Deficiency or loss of EPC's can interrupt the balance between endothelial damage and restorative function, leading to endothelial dysfunction, atherosclerosis, and elevated vascular risk. In addition, increased levels of prothrombin, von Willebrand factor, and endothelin also increase cardiovascular risk.

\section{Environmental Factors}

Many studies have clearly shown that a change in environmental factors often triggers migraine attacks. A large systemic review has established that the most common triggers for migraine attacks are stress, auditory stimuli, fatigue, fasting, weather changes, and menses [9].

Some studies suggest that increased risk of cardiovascular events in migraine patients can be ascribed to a higher occurrence of cardiovascular risk factors, such as smoking, alcohol, high salt intake (hypertension) and obesity (hypercholesterolemia) [26]. Smoking, especially in women migraine patients, increases the risk of stroke [33]. Advice on healthy lifestyle, mainly smoking cessation, control of hypertension (HTN), and maintenance of normal body weight, may aid in controlling some of the modifiable risk factors.

If we control these modifiable cardiovascular risk factors in migraine patients, will they still experience the cardiovascular events?

\section{Genetic Factors}

If migraine and coronary artery disease (CAD) share the same pathological process, they likely share genetic risk as well. Winsvold et al. determined the genetic overlap between migraine and CAD by performing analyses based on three large genome-wide association study (GWAS) meta-analyses of migraine and coronary artery disease [23]. One shared risk locus was identified as encoding phosphatase and actin regulator 1 (PHACTR1) gene. This is a genome-wide significant risk locus for both migraine and CAD. This protein phosphatase 1 binding protein is highly expressed in the brain where it regulates synaptic activity and dendritic morphology. It is also expressed in arteries and plays an important role in the regulation of endothelial function and is associated with altered vasomotor tone [34].

More work needs to be done in order to improve our understanding of the genetic association between migraine and CAD. More importantly, how this genetic association plays a role in the pathogenic and vascular mechanism of migraine? Is there more than one gene involved in this association? 
Sex hormones seem to be related to migraine, and this may be responsible for the gender difference seen in migraine patients. Fluctuating levels of sex hormones, especially estrogen, predisposes females to migraine attacks by increasing cortical excitability. Estrogen has a major influence on vascular health as it is involved in both thrombotic as well as vasodilatory mechanisms. However, the role of estrogen in causing IHD in migraine patients remains unclear because of the complex mechanisms involved [27,35].

Recently, the European Headache Federation (EHF) and the European Society of Contraception and Reproductive Health (ESC) has published a statement regarding the use of hormonal contraceptives in female migraineurs [36]. In particular, the combination of smoking and oral contraceptives should be avoided in all cases in young women with migraine (aura) as this increases the risk for ischemic stroke [33].

Although the American Heart Association (AHA) has also suggested practicing caution when prescribing oral contraceptive pills (OCP), there is inadequate evidence for this recommendation [37].

Currently, there is not enough evidence to support changes in medication that may decrease the cardiovascular risk of migraine in women. Further studies should find the exact mechanism behind the gender difference in CVD risk in migraine patients.

\section{Miscellaneous}

The mechanism by which migraine increases CVD risk is multi-factorial. Different mechanisms are involved between migraine and different cardiovascular outcomes. A number of studies have shown that the patency of foramen ovale (PFO) is higher in migraine with aura which can lead to paradoxical emboli reaching the cerebral or coronary vasculature causing a stroke or myocardial infarction respectively [18]. Some clinical trials have shown that the closure of PFO can reduce the risk of stroke, but the PFO closure for migraine headache remains controversial.

Migraine patients often use non-steroid anti-inflammatory drugs (NSAIDs), which is suspected to be associated with a higher risk of myocardial infarction, venous thromboembolism (VTE) and atrial fibrillation $[19,21,22]$. Although currents guidelines do not recommend the use of aspirin and clopidogrel in the prophylaxis of migraine, their efficacy in preventing migraine is still under study [38].

Physicians should be aware of this association and should prescribe NSAIDs with caution, especially in patients who have a higher risk of VTE. In addition, immobilization due to migraine attacks can further increase the likelihood of VTE.

Should the physicians stop prescribing NSAIDS to migraine patients because of their cardiovascular risk profile?

What is new on the horizon?

- What mechanisms other than those proposed by previous studies are inflicting the association between migraine headache and IHD?

- If we manage the cardiovascular risk factor in migraine patients, are they still likely to experience cardiovascular events?

- The literature supports the proposal that migraine patients experience cardiovascular changes. Can it be the other way round too? Can migraine also occur in a patient with a history of IHD?

- Careful lifestyle advice like maintaining ideal body weight and smoking cessation should be given to patients who are at risk of developing CVD.

- More work must be done, to enhance our understanding of the genetic association between migraine headache and CVD.

- Researchers need to find out if any changes in medication can be beneficial for migraine patients.

Limitations

Although most of the articles used in our review were of high quality, there were some limitations that we faced. Firstly, we did not have full access to all the articles as some of the literature was paid. Secondly, not all articles were in the English language, so we faced a language barrier during our research. Thirdly, we could not access all the research engines as they were available only to members. Fourth, some of the studies 
have shown heterogeneity in results that should not be ignored. Lastly, we did not consider the association between migraine and other vascular disorders, which has been mentioned in some of the studies.

\section{Conclusions}

Based on our research, we found out that migraine headache is linked with IHD. Migraine with aura is, in fact, strongly associated with adverse cardiovascular outcomes such as myocardial infarction, angina, and arrhythmia. Migraine, therefore, should be considered as an overall risk factor for cardiovascular disease. Physicians should educate the patients regarding this association and advise lifestyle modification to reduce this risk.

\section{Additional Information \\ Disclosures}

Conflicts of interest: In compliance with the ICMJE uniform disclosure form, all authors declare the following: Payment/services info: All authors have declared that no financial support was received from any organization for the submitted work. Financial relationships: All authors have declared that they have no financial relationships at present or within the previous three years with any organizations that might have an interest in the submitted work. Other relationships: All authors have declared that there are no other relationships or activities that could appear to have influenced the submitted work.

\section{References}

1. Headache disorders. (2016). http://www.who.int/mediacentre/factsheets/fs277/en/.

2. Migraine facts. Accessed: February 21, 2018: http:// http://migraineresearchfoundation.org/aboutmigraine/migraine-facts.

3. Headache Classification Committee of the International Headache Society (IHS): The international classification of headache disorders, 3rd edition (beta version). Cephalalgia. 2013, 33:629-808. $10.1177 / 0333102413485658$

4. GBD 2015 Disease and Injury Incidence and Prevalence Collaborators: Global, regional, and national incidence, prevalence, and years lived with disability for 310 diseases and injuries, 1990-2015: a systematic analysis for the Global Burden of Disease Study 2015. Lancet. 2016, 388:1545-602. 10.1016/S01406736(16)31678-6

5. The facts about migraine. (2019). https://americanmigrainefoundation.org/resource-library/facts-aboutmigraine/.

6. Tepper D: Migraine and cardiovascular disease. Headache. 2014, 54:1267-8. 10.1111/head.12410

7. Alqaqa A: The association of cardiovascular disease and migraine: review . J Clini Exp Cardiol. 2016, 7:465. 10.4172/2155-9880.1000465

8. Winsvold BS, Bettella F, Witoelar A, et al.: Shared genetic risk between migraine and coronary artery disease: a genome-wide analysis of common variants. PLoS One. 2017, 12:e185663. 10.1371/iournal.pone. 0185663

9. Marmura MJ: Triggers, protectors, and predictors in episodic migraine. Curr Pain Headache Rep. 2018, 22:81. 10.1007/s11916-018-0734-0

10. Bektas H, Karabulut H, Doganay B, Acar B: Allergens might trigger migraine attacks . Acta Neurologica Belgica. 2017, 117:91-95. 10.1007/s13760-016-0645-y

11. Spector JT, Kahn SR, Jones MR, Jayakumar M, Dalal D, Nazarian S: Migraine headache and ischemic stroke risk: an updated meta-analysis. Am J Med. 2010, 123:612-24. 10.1016/j.amjmed.2009.12.021

12. Kurth T, Chabriat H, Bousser MG: Migraine and stroke: a complex association with clinical implications . Lancet Neurol. 2012, 11:92-100. 10.1016/S1474-4422(11)70266-6

13. Diener H, Kurth T, Holle D: Practical implications of the migraine cardio- and cerebrovascular association: unmet needs of patients. Cephalalgia. 2015, 35:140-5. 10.1177/0333102414554662

14. Adelborg K, Szépligeti S, Holland-Bill L, et al.: Migraine and risk of cardiovascular diseases: Danish population based matched cohort study. BMJ. 2018, 360:k96. 10.1136/bmj.k96

15. Sacco S, Ornello R, Ripa P, Tiseo C, Degan D, Pistoia F, Carolei A: Migraine and risk of ischaemic heart disease: a systematic review and meta-analysis of observational studies. Eur J Neurol. 2015, 24:1001-1011. 10.1111/ene.12701

16. Rambarat C, Elgendy I, Johnson B, et al.: Migraine headache and long-term cardiovascular outcomes: an extended follow-up of the women's ischemia syndrome evaluation. Am J Med. 2017, 130:738-743. 10.1016/j.amjmed.2016.12.028

17. Butt JH, Franzmann U, Kruuse C: Endothelial function in migraine with aura - a systematic review . Headache. 2015, 55:35-54. 10.1111/head.12494

18. Schwerzmann M, Nedeltchev K, Lagger F, Mattle HP, Windecker S, Meier B, Seiler C: Prevalence and size of directly detected patent foramen ovale in migraine with aura. Neurology. 2005, 65:1415-8. 10.1212/01.wnl.0000179800.73706.20

19. Trelle S, Reichenbach S, Wandel S, et al.: Cardiovascular safety of non-steroidal anti-inflammatory drugs: network meta-analysis. BMJ. 2011, 342:c7086. 10.1136/bmj.c7086

20. Kurth T, Diener HC, Buring JE: Migraine and cardiovascular disease in women and the role of aspirin: subgroup analyses in the Women's Health Study. Cephalalgia. 2011, 31:1106-15. 10.1177/0333102411412628

21. Ungprasert P, Srivali N, Wijarnpreecha K, Charoenpong P, Knight EL: Non-steroidal anti-inflammatory drugs and risk of venous thromboembolism: a systematic review and meta-analysis. Rheumatology. 2015 , 54:736-42. 10.1093/rheumatology/keu408 
22. Schmidt M, Christiansen CF, Mehnert F, Rothman KJ, Sørensen HT: Non-steroidal anti-inflammatory drug use and risk of atrial fibrillation or flutter: population based case-control study. BMJ. 2011, 343:3450. 10.1136/bmj.d3450

23. Winsvold BS, Bettella F, Witoelar A, et al.: Shared genetic risk between migraine and coronary artery disease: a genome-wide analysis of common variants. PLoS One. 2017, 12:e0185663. 10.1371/journal.pone.0185663

24. Winsvold BS, Nelson CP, Malik R, et al.: Genetic analysis for a shared biological basis between migraine and coronary artery disease. Neurol Genet. 2015, 1:e10. 10.1212/NXG.0000000000000010

25. Pickrell JK, Berisa T, Liu JZ, Segurel L, Tung JY, Hinds DA: Detection and interpretation of shared genetic influences on 42 human traits. Nat Genet. 2016, 48:709-17. 10.1038/ng.3570

26. Mahmoud A, Mentias A, Elgendy A, et al.: Migraine and the risk of cardiovascular and cerebrovascular events: a meta-analysis of 16 cohort studies including 1152407 subjects. BMJ Open. 2018, 8:e020498. 10.1136/bmjopen-2017-020498

27. Linstra K, Ibrahimi K, Terwindt G, et al.: Migraine and cardiovascular disease in women . Maturitas. 2017, 97:28-31. 10.1016/j.maturitas.2016.12.008

28. Kurth T, Winter A, Eliassen A, et al.: Migraine and risk of cardiovascular disease in women: prospective cohort study. BMJ. 2016, 353:i2610. 10.1136/bmj.i2610

29. Åsberg A, Stovner L, Zwart J, Winsvold BS, Heuch I, Hagen K: Migraine as a predictor of mortality: the HUNT study. Cephalalgia. 2015, 36:351-7. 10.1177/0333102415593090

30. Schürks M, Rist P, Shapiro R. Kurth T: Migraine and mortality: a systematic review and meta-analysis . Cephalalgia. 2011, 31:1301-1314. 10.1177/0333102411415879

31. Wang YC, Lin CW, Ho YT, Huang YP, Pan SL: Increased risk of ischemic heart disease in young patients with migraine: a population-based, propensity score-matched, longitudinal follow-up study. Int J Cardiol. 2014, 172:213-6. 10.1016/j.ijcard.2014.01.005

32. Bernecker C, Ragginer C, Fauler G, et al.: Oxidative stress is associated with migraine and migraine related metabolic risk in females. Eur J Nourol. 2011, 18:1233-9. 10.1111/j.1468-1331.2011.03414.x

33. Al-Hassany L, Linstra KA, Terwindt GM. van den Brink AM: Cardiovascular risk of migraine in men and women. Gender and Migraine. Headache. van den Brink AM, MacGregor E (ed): Springer, Cham, Switzerland; 2019. 17-29. 10.1007/978-3-030-02988-3 2

34. Allen PB, Greenfield AT, Svenningsson P, Haspeslagh DC, Greengard P: Phactrs 1-4: a family of protein phosphatase 1 and actin regulatory proteins. PNAS. 2004, 101:7187-92. 10.1073/pnas.0401673101

35. Chai NC, Peterlin BL, Calhoun AH: Migraine and estrogen. Curr Opin Neurol. 2014, 27:315-324. 10.1097/WCO.0000000000000091

36. Sacco S, Merki-Feld GS, Ægidius KL, et al.: Hormonal contraceptives and risk of ischemic stroke in women with migraine: a consensus statement from the European Headache Federation (EHF) and the European Society of Contraception and Reproductive Health (ESC). J Headache Pain. 2017, 18:108. 10.1186/s10194017-0815-1

37. Bushnell C, McCullough LD, Awad IA, et al.: Guidelines for the prevention of stroke in women: a statement for healthcare professionals from the American Heart Association/American Stroke Association. Stroke Association. 2014, 45:1545-1588. 10.1161/01.str.0000442009.06663.48

38. Headaches in over 12s: diagnosis and management. (2015). http://www.nice.org.uk/guidance/cg150/chapter/recommendations. 